\title{
Improved Transfer Process for the Fully Additive Manufacturing of a Conductive Layer-Stacked Polymeric Cantilever
}

\author{
Shusuke Kanazawa*, Yasuyuki Kusaka, Noritaka Yamamoto, Hirobumi Ushijima \\ Flexible Electronics Research Center, National Institute of Advanced Industrial Science and Technology, Tsukuba, Japan \\ Email: *kanazawa-s@aist.go.jp
}

How to cite this paper: Kanazawa, S., Kusaka, Y., Yamamoto, N. and Ushijima, H. (2019) Improved Transfer Process for the Fully Additive Manufacturing of a Conductive Layer-Stacked Polymeric Cantilever. Materials Sciences and Applications, 10, 45-52.

https://doi.org/10.4236/msa.2019.101005

Received: November 23, 2018

Accepted: January 11, 2019

Published: January 14, 2019

Copyright $\odot 2019$ by author(s) and Scientific Research Publishing Inc. This work is licensed under the Creative Commons Attribution International License (CC BY 4.0).

http://creativecommons.org/licenses/by/4.0/

\begin{abstract}
This paper reports on an efficient fabrication process for a polymeric cantilever covered with conductive nano silver. The entire structure can be constructed additively using a printing process, without the use of an etching process or a sacrificial layer. The fabricated cantilever exhibits good linearity and forms a submillimeter-ordered air gap between itself and the substrate surface. Fine operation of a capacitive force gauge was obtained using the capacitance between the conductive cantilever and an electrode on the substrate. This process is expected to make possible the efficient manufacturing of various types of sensors that measure mechanical strain in a cantilever structure.
\end{abstract}

\section{Keywords}

Additive Manufacturing, MEMS, Screen Printing, Polymer, Sensor

\section{Introduction}

Sensor devices are attracting increasing attention because they are expected to be able to play an important role in the internet of things. Highly improved sensors that can detect the condition of humans [1] [2], objects [3], and the environment [4] have been proposed. This progress indicates that a large number of sensors will be produced in the near future to help us in every aspect of our lives. In fact, it has been predicted that the production of sensors will reach one million per year [5]. In the foreseeable future, it will be important to have highly efficient manufacturing processes for sensors to ensure social sustainability.

Hollowed structures such as cantilevers and bridges are used frequently to fabricate sensor devices [6]-[12]. Such structures can perform the core function of 
sensors by means of the mechanical strain against the object to be detected. However, the fabrication processes for such structures include several steps and result in waste of materials, particularly the sacrificial layer. The increase in production of sensors makes it necessary to develop a novel method for obtaining hollowed structures in a simpler way. We have developed a printing-based method for fabrication of cantilevers without the use of a sacrificial layer [13] [14]. In a previous study, an Ag paste that was printed and sintered on a temporary substrate was transferred to the head of a step on a substrate to produce a conductive cantilever. Although the model for this fabrication procedure has been established, it needs to be applied to various materials before the process can become widely used. In this paper, an improved process for fabrication of a conductive cantilever using a resin paste is reported. The process involves the deposition of a conductive layer on the resin and the subsequent transfer of the conductive layer and resin as a batch to form a cantilever. It is expected that this improved process will make it possible to manufacture various types of polymer cantilevers that can be used in a variety of electronics applications, such as sensors.

\section{Experimental Methods and Materials}

\subsection{Forming the Pre-Structure of the Cantilever}

This section corresponds to (a) and (b) in Figure 1, which illustrates the flow of

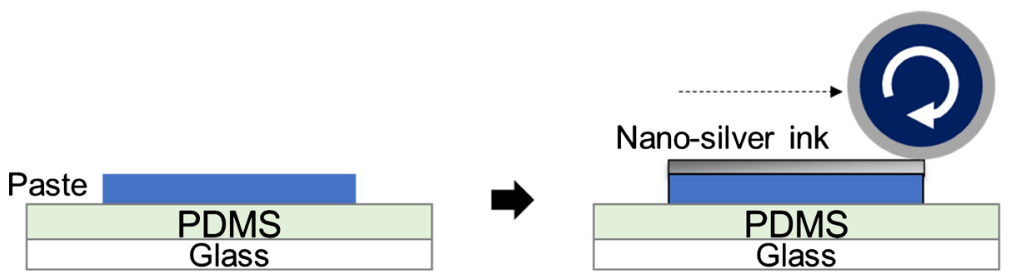

(a)

(b)

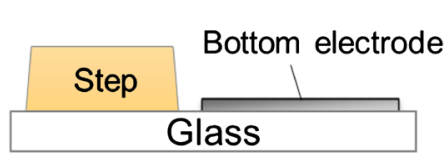

(c)

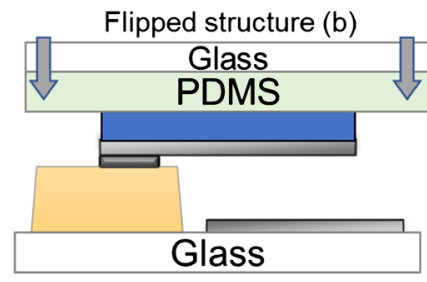

(e)

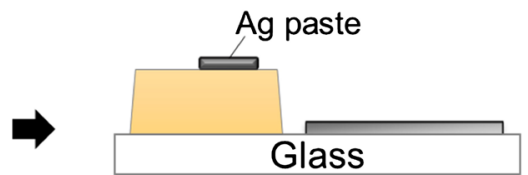

(d)

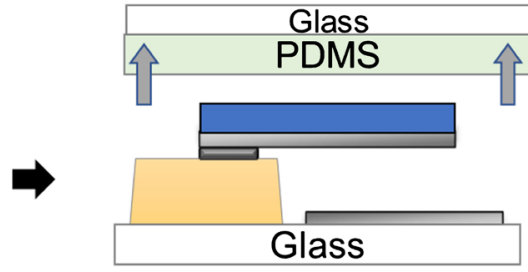

(f)

Figure 1. Process flow for producing a polymer cantilever with a conductive layer. 
the process schematically. An elastomer of poly(dimethylsiloxane) (PDMS) (Shin-Etsu Chemical, KE-106) on a base glass plate was used as a temporary substrate. The resin that was to form the base of the cantilever was screen printed on the PDMS using a metal mask. A $100-\mu \mathrm{m}$-thick metal mask with the openings of the cantilever design was laid on the PDMS. The cantilever design is described in Section 3.1. The metal mask was fixed onto the PDMS simply by being placed onto it. A paste of photo-curable resin used for screen printing (GOO Chemical) was then coated onto the surface of the metal mask surface using a squeegee to apply the paste on the PDMS through the opening, as shown in Figure 1(a). When the metal mask was removed, the PDMS surface had a layer of paste, which was patterned according to the openings of the mask. To sinter the paste, ultraviolet (UV) exposure was performed using a high-pressure mercury lamp at 500 $\mathrm{mJ} / \mathrm{cm}^{2}$.

To deposit a conductive layer onto the sintered resin, micro-contact printing [15] was performed using a nano silver ink (DIC, RAGT-19), as illustrated in Figure 1(b). Micro-contact printing was used to pattern inks based on differences in height, in micrometer ranges. The ink was selectively received based on the height of the resin on the PDMS. The nano silver ink was first coated onto a silicon blanket (Kinyosha) attached to a printing device (Nihon Denshi Seiki) and then deposited onto the resin. After printing, the nano silver ink was thermally sintered at $150^{\circ} \mathrm{C}$ for $30 \mathrm{~min}$ to cure it.

\subsection{Preparation of the Substrate with a Step and a Bottom Electrode}

The substrate that received the preformed structure on the PDMS was prepared using a glass plate, as shown in Figure 1(c) and Figure 1(d). In this study, we used the cantilever as a capacitive force gauge, so that the bottom electrode was not formed on the substrate. This was achieved through reverse offset printing, using the same nano silver ink used for the micro contact printing [16]. A photoresist (Nippon Kayaku, SU-8 25) was used to form an insulator step beside the bottom electrode. The photoresist was spin-coated at $2000 \mathrm{rpm}$ and dried at $110^{\circ} \mathrm{C}$ for $10 \mathrm{~min}$ on a hot plate. After UV exposure at $130 \mathrm{~mJ} / \mathrm{cm}^{2}$ through a photomask, a post etching bake was conducted at $110^{\circ} \mathrm{C}$ for $10 \mathrm{~min}$, and the photoresist was subsequently developed for $15 \mathrm{~min}$ and dried to obtain the structure shown in Figure 1(c).

An adhesive point was provided on the top surface of the step to receive the cantilever. As Figure 1(d) shows, it was formed by screen printing using a conductive Ag paste (DIC, GOAGT-93C) with dimensions of $1 \mathrm{~mm} \times 1 \mathrm{~mm}$. We proceeded to the next step without sintering the Ag paste to maintain its adhesiveness.

\subsection{Transfer of the Cantilever}

As mentioned previously, a pre-structure of the cantilever on a temporary substrate and a substrate to receive the cantilever were prepared. The preformed 
cantilever, shown in Figure 1(b), was made to contact the step on the substrate vertically. As Figure 1(e) shows, the pre-structure was laid on the adhesive point on the step. While the two were contact, a pressure of $3.1 \mathrm{kPa}$ was applied, and the substrates were annealed at $150^{\circ} \mathrm{C}$ for $1 \mathrm{~h}$. Finally, the two substrates were vertically peeled off to transfer the cantilever. The structure obtained was examined using a scanning electron microscope (SEM, KEYENCE, D-500) and a laser microscope (Laser tech, OPTELICS).

\subsection{Operation as a Capacitive Force Gauge}

As a result of the process described above, an air gap capacitance was formed between the bottom electrode and the back surface of the cantilever. The performance of the structure as a capacitive force gauge was then evaluated. To extend the conduction from the back surface of the cantilever, a conductive paste (Fujikura Kasei, D-550) was dropped at the base edge of the cantilever and then connected to an inductance (L)-capacitance (C)-resistance (R) (LCR) meter (HIOKI E. E., IM3590). The capacitance was measured at a voltage of $5 \mathrm{~V}$ and frequency of $10 \mathrm{kHz}$. Although the initial capacitance was evaluated in a shield box, the other measurements were performed without shielding by applying forces of various magnitudes to the cantilever. Forces of $1-200 \mu \mathrm{N}$ were applied vertically to the top surface of the cantilever using a contact-type thickness meter (Kosaka laboratory, ET4000). The thickness meter was also used to measure the z-position of the cantilever. The capacitances and z-positions for each level of applied force were measured by the system.

\section{Result and Discussion}

\subsection{Result of the Pre-Structure Fabrication}

The pre-structures of the cantilever are shown in Figure 2. Figure 2(a) shows the patterns of the resin for the pre-structures, and Figure 2(b) shows the pre-structures after the micro contact with nano silver ink. For the design of the cantilevers, we used a combination of a square and a rectangle. The size of the square $(3 \mathrm{~mm} \times 3 \mathrm{~mm})$ was the same for all the cantilevers. The width of the rectangle varied from $0.75 \mathrm{~mm}$ to $2.0 \mathrm{~mm}$, and the length varied from $5.0 \mathrm{~mm}$ to

(a)

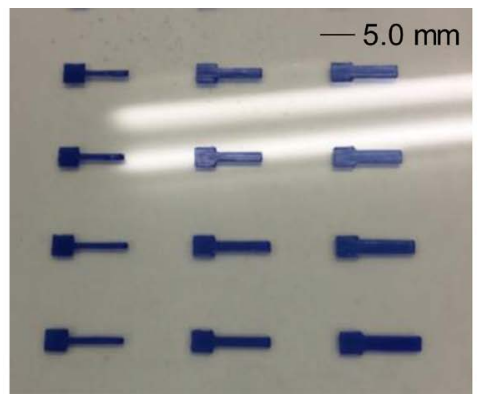

(b)

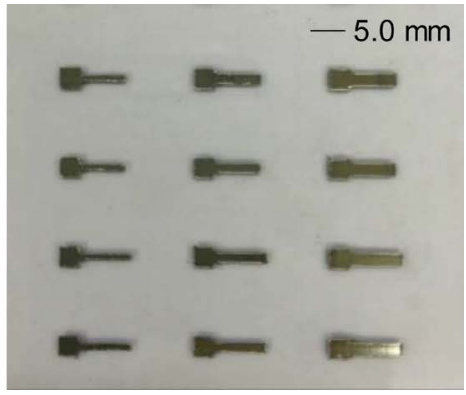

Figure 2. Appearance of the pre-structure of the cantilever. (a) Patterned resin prepared by the Step (a) in the Figure 1. (b) Nano silver ink-stacked resin prepared by the Step (b) in the Figure 1. 
$7.0 \mathrm{~mm}$. These shapes followed the pattern of the openings in the metal mask accurately. Although we used a millimeter range for the design in this study, it has been reported that fine lines of $30 \mu \mathrm{m}$ [17] can be screen-printed on PDMS. Therefore, our method is considered to also be suitable for precise printing. The thickness of the resin was typically $78 \mu \mathrm{m}$, independent of the size of the cantilever. After the micro contact, a 220-nm-thick nano silver layer was deposited on the resin. A comparison of Figure 1(a) and Figure 1(b) shows that the glossy surface of the nano silver ink was applied only to the resin. Because of the $78-\mu \mathrm{m}$ thickness of the resin, the nano silver ink was selectively applied by the micro contact printing.

\subsection{Appearance of the Fabricated Cantilever}

An SEM image of a fabricated cantilever with a width of $0.75 \mathrm{~mm}$ and a length of $7.0 \mathrm{~mm}$ is shown in Figure 3. It can be seen that it is fixed on the step and forms a hollowed part toward the top edge. The thickness of the step is $45 \mu \mathrm{m}$. The bright area under the hollowed part is the bottom electrode, which overlaps the cantilever for a length of $5.8 \mathrm{~mm}$. The pre-formed structure was completely transferred to the substrate from the PDMS surface without any structural defect. No residue was observed on the PDMS by the SEM after the transfer.

To evaluate the linearity of the fabricated cantilever, we measured the height of the upper surface from the substrate surface (or the bottom electrode surface, to be exact) at several points, as shown in Figure 4. The filled points ( ) correspond to the upper surface. As the typical thickness of the cantilever was 78 $\mu \mathrm{m}$, the air gap between the substrate and the back surface can be estimated from the open points $(\bigcirc)$. The estimated profile suggest that the cantilever had a slight tilt toward the top edge. The tilt angle was calculated to be $2.4 \times 10^{-3}$. This small tilt indicates that a cantilever with good linearity can be fabricated by this process.

\subsection{Capacitive Force Gauge Characteristics of the Fabricated Cantilever}

The initial capacitance measured in the shield box was $0.73 \mathrm{pF}$. The theoretical capacitance can be calculated using Equation (1).

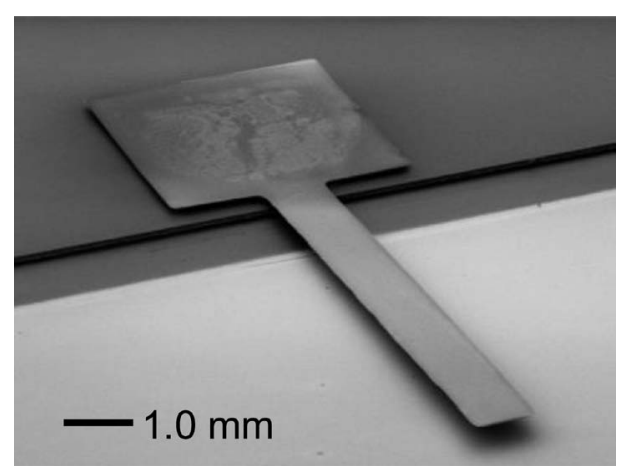

Figure 3. SEM image of a fabricated cantilever. 


$$
C=\varepsilon_{0} \varepsilon_{r} S / d .
$$

In this equation, $C, \varepsilon_{0}, \varepsilon_{r}, S$, and $d$ are the capacitance, permittivity of the vacuum, relative permittivity, area of capacitance, and length of the air gap, respectively. We used the average estimated gap between the back surface and the substrate, as shown in Figure 4, as the temporary air gap. The theoretical capacitance was determined to be $0.75 \mathrm{pF}$, which is close to the measured value. This agreement indicates that the capacitance between the cantilever and the substrate formed correctly. It also indicates that the hollowed part of the cantilever achieved conductivity by the addition of the nano silver ink that forms the back surface.

Figure 5 shows the operation of the capacitive force gauge. The z-position originates at $1 \mu \mathrm{N}$, and the capacitances were normalized by the initial results. It can be seen that there is a change in capacitance corresponding to the z-position with the application of minute forces in the micronewton range. A clear operation was believed to have been achieved because of the mechanical flexibility of the cantilever, which consists mainly of the resin. We believe that such a structure, constructed of polymers, can be used in the detection of a wide variety of

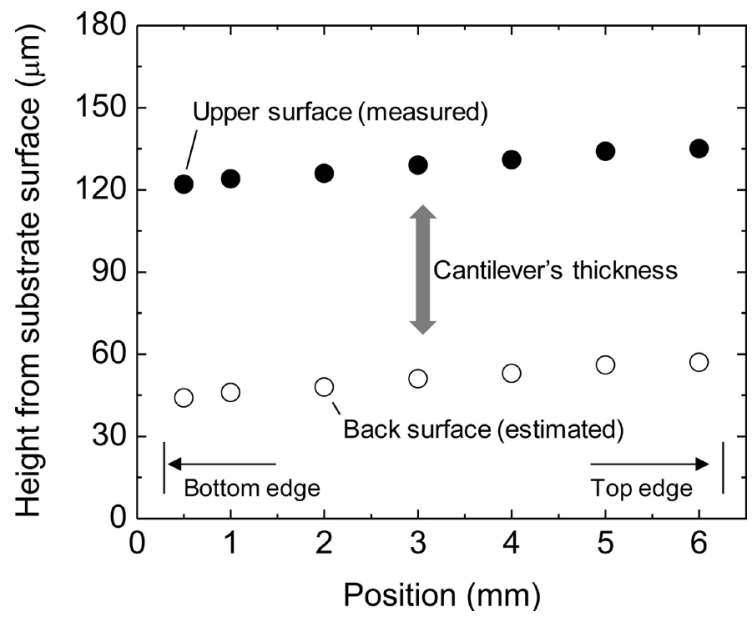

Figure 4. Estimated profile of the fabricated cantilever.

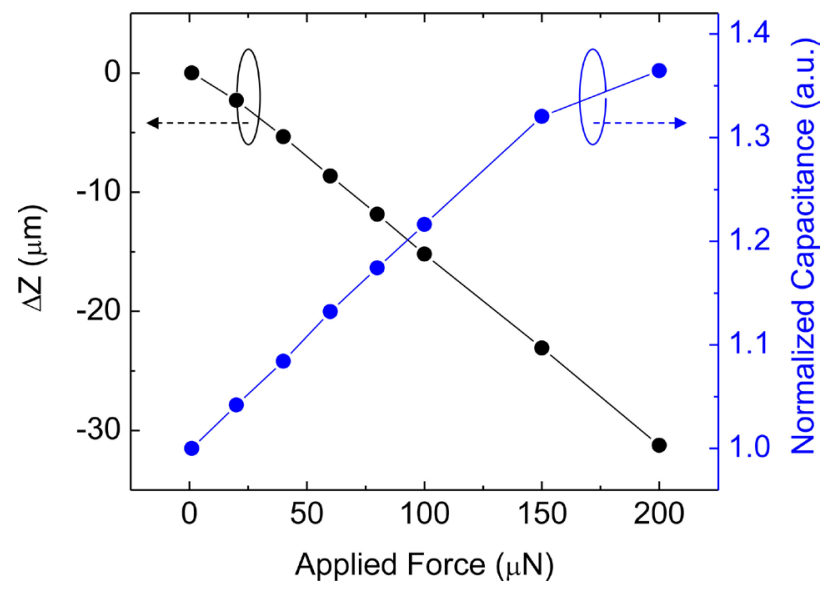

Figure 5. Capacitive force gauge operation of the fabricated cantilever. 
minute phenomena. We also believe that the process described here can be used to manufacture various types of hollowed structures efficiently using polymer materials.

\section{Conclusion}

We developed an improved process for fabricating a conductive cantilever using a resin. This highly efficient process involves a combination of printing and transfer. The fabricated cantilever exhibited good linearity, which reflects the usefulness of the process. In addition, a force gauge operation was demonstrated against loads in the micronewton range. These results demonstrate that hollowed structures based on polymers can be fabricated efficiently using the process developed in this study.

\section{Fund}

This work was supported by the Japan Society for the Promotion of Science (JSPS) KAKENHI Grant Number 17K18419.

\section{Conflicts of Interest}

The authors declare no conflicts of interest regarding the publication of this paper.

\section{References}

[1] Gao, W., Emaminejad, S., Nyein, H.Y.Y., Challa, S., Chen, K., Peck, A., Fahad, H.M., Ota, H., Shiraki, H., Kiriya, D., Lien, D-H., Brooks, G.A., Davis, R.W. and Javey, A. (2016) Fully Integrated Wearable Sensor Arrays for Multiplexed in Situ Perspiration Analysis. Nature, 529, 509-514. https://doi.org/10.1038/nature16521

[2] Gong, S., Schwalb, W., Wang, Y., Chen, Y., Tang, Y., Si, J., Shirinzadeh, B. and Cheng, W. (2015) A Wearable and Highly Sensitive Pressure Sensor with Ultrathin Gold Nanowires. Nature Communications, 5.

[3] Pan, L., Chortos, A., Yu, G., Wang, Y., Isaacson, S., Allen, R., Shi, Y., Dauskardt, R., and Bao, Z. (2014) An Ultra-Sensitive Resistive Pressure Sensor Based on Hollow-Sphere Microstructure Induced Elasticity in Conducting Polymer Film. Nature Communications, 5.

[4] Knopfmacher, O., Hammock, M.L., Appleton, A.L., Schwartz, G., Mei, J., Lei, T., Pei, J. and Bao, Z. (2014) Highly Stable Organic Polymer Field-Effect Transistor Sensor for Selective Detection in the Marine Environment. Nature Communications, 5.

[5] Bogue, R. (2014) Towards the Trillion Sensors Market. Sensor Review, 34, 137-142. https://doi.org/10.1108/SR-12-2013-755

[6] Wu, C., Petrini, V., Joseph, E. and Amiot, F. (2014) Design and Fabrication of a Multiple-Thickness Electrochemical Cantilever Sensor. Microelectronic Engineering, 119, 1-5. https://doi.org/10.1016/j.mee.2014.01.009

[7] Johansson, A., Calleja, M., Rasmussen, P.A. and Boisen, A. (2005) SU-8 Cantilever Sensor System with Integrated Readout. Sensors and Actuators A, 123-124, 111-115. https://doi.org/10.1016/j.sna.2005.03.025

[8] Wasisto, H.S., Merzsch, S., Waag, A., Uhde, E., Salthammer, T. and Peiner, E. 
(2012) Effect of Photoresist Coating on the Reusable Resonant Cantilever Sensors for Assessing Exposure to Airborne Nanoparticles. Sensors and Actuators A, 47, 302-305.

[9] Sharma, H. and Mutharasan, R. (2013) Rapid and Sensitive Immunodetection of Listeria monocytogenes in Milk Using a Novel Piezoelectric Cantilever Sensor. Biosensors and Bioelectronics, 45, 158-162. https://doi.org/10.1016/j.bios.2013.01.068

[10] Sharma, H. and Mutharasan, R. (2013) hlyA Gene-Based Sensitive Detection of Listeria monocytogenes Using a Novel Cantilever Sensor. Analytical Chemistry, 85, 3222-3228. https://doi.org/10.1021/ac303561c

[11] Hou, H., Bai, X., Xing, C., Gu, N., Zhang, B. and Tang, J. (2013) Aptamer-Based Cantilever Array Sensors for Oxytetracycline Detection. Analytical Chemistry, 85, 2010-2014. https://doi.org/10.1021/ac3037574

[12] Gammelgaard, L., Rasmussen, P.A., Calleja, M., Vettiger, P. and Boisen, A. (2006) Microfabricated Photoplastic Cantilever with Integrated Photoplastic/Carbon Based Piezoresistive Strain Sensor. Applied Physics Letters, 88, Article ID: 113508. https://doi.org/10.1063/1.2186396

[13] Kanazawa, S., Kusaka, Y., Yamamoto, N. and Ushijima, H. (2017) Novel Printing Process for the Fabrication of Cantilever Structures by the Partially Controlled Sintering of Ink. Japanese Journal of Applied Physics, 56, Article ID: 010313.

[14] Kanazawa, S., Kusaka, Y., Yamamoto, N. and Ushijima, H. (2017) Improved Transfer Process for Fabrication of Cantilever with Precise Air-Gap Formation. Japanese Journal of Applied Physics, 56, 05EB01.

[15] Kina, O., Koutake, M., Matsuoka, K. and Yase, K. (2010) Organic Thin-Film Transistors Fabricated by Microcontact Printing. Japanese Journal of Applied Physics, 49, 01AB07. https://doi.org/10.1143/JJAP.49.01AB07

[16] Kusaka, Y., Sugihara, K., Koutake, M. and Ushijima, H. (2014) Fabrication of Embedded Electrodes by Reverse Offset Printing. Journal of Micromechanics and Microengineering, 24, Article ID: 035020. https://doi.org/10.1088/0960-1317/24/3/035020

[17] Nomura, K., Ushijima, H., Mitsui, R., Takahashi, S. and Nakajima, S. (2014) Continuous Fine Pattern Formation by Screen-Offset Printing Using a Silicone Blanket. Microelectronic Engineering, 123, Article ID: 095021. https://doi.org/10.1016/j.mee.2014.05.009 